
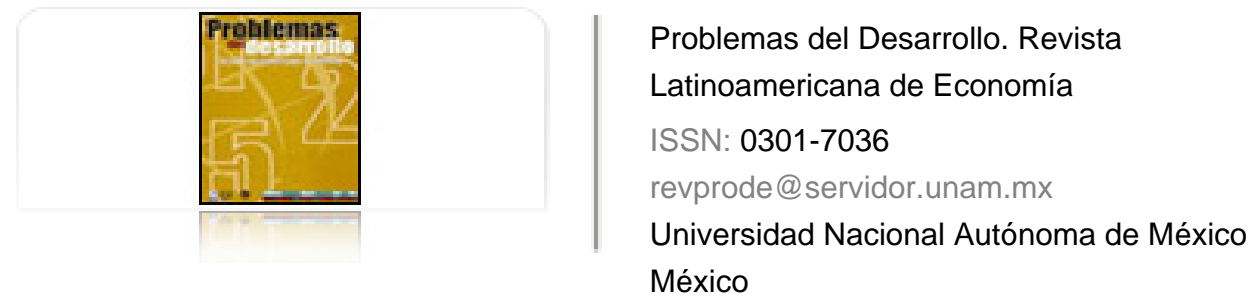

Reza, Germán A. de la

La dimensión entrópica del sector externo en las economías abiertas

Problemas del Desarrollo. Revista Latinoamericana de Economía, vol. 39, núm. 152, enero-marzo, 2008, pp. 69-86

Universidad Nacional Autónoma de México

Distrito Federal, México

Disponible en: http://www.redalyc.org/articulo.oa?id=11820668005

Cómo citar el artículo

Número completo

- Más información del artículo

Página de la revista en redalyc.org

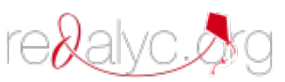

Sistema de Información Científica

Red de Revistas Científicas de América Latina, el Caribe, España y Portugal Proyecto académico sin fines de lucro, desarrollado bajo la iniciativa de acceso abierto 


\title{
LA DIMENSIÓN ENTRÓPICA DEL SECTOR EXTERNO EN LAS ECONOMÍAS ABIERTAS
}

Germán A. de la Reza*

\begin{abstract}
Fecha de recepción: 8 de octubre de 2007. Fecha de aceptación: 20 de noviembre de 2007.
\end{abstract}

\section{Resumen}

En el presente artículo se analiza la dimensión entrópica del sector externo de una economía nacional entendida como sistema abierto. El trabajo tiene por objetivo la normativización del estudio entrópico para la explicación de procesos económicos. Con ese fin propone un constructo cognitivo basado en la teoría de sistemas y cuyas características buscan superar el estancamiento del diálogo interdisciplinario y asociar de manera estrecha los niveles micro y macroeconómicos.

Palabras clave: entropía, sector externo, sistemas complejos, análisis multivariable, feedback.

* Profesor-investigador del Departamento de Economía, UAM-Azcapotzalco; investigador del Sistema Nacional de Investigadores (SNI). El autor desea agradecer las observaciones y correcciones sugeridas por dos dictaminadores anónimos. Correo electrónico: delareza@ hotmail.com. 
70 GERMÁN A. DE LA REZA

\section{Summary}

This article analyzes the entropic dimension of the external sector of a national economy understood as an open system. The work aims to normalize the entropic study to explain economic processes. With this end, it proposes a cognitive construct based on systems theory, characterized by an attempt to overcome the stagnation in interdisciplinary dialogue and making a close association between the microeconomic and macroeconomic levels.

Key words: entropy, external sector, complex systems, multivariate analysis, feedback.

\section{Résumé}

Dans cet article, il est procédé à l'analyse de l'entropie du secteur externe d'une économie nationale vue comme un système ouvert. Ce travail a pour objectif la normalisation de l'étude de l'entropie pour l'explication de processus économiques. Dans ce but il propose une construction cognitive basée sur la théorie des systèmes qui vise à dépasser l'enlisement du dialogue interdisciplinaire et associer étroitement les plans micro et macro-économique.

Mots clés: entropie, secteur externe, systèmes complexes, analyse multivariable, feedback.

\section{Resumo}

No presente artigo analisa-se a dimensão entrópica do sector externo de uma economia nacional entendida como sistema aberto. $O$ trabalho tem por objetivo a normativização do estudo entrópico para a explicação de processos econômicos. Com esse fim propõe um construto cognitivo baseado na teoria de sistemas e cujas características buscam superar o estancamento do diálogo interdisciplinario e associar de maneira estreita os níveis micro e macroeconômicos.

Palavras-chave: entropia, setor externo, sistemas complexos, análise multivariável, feedback. 


\section{Introducción}

X

edio siglo después de los primeros ensayos de generalización del principio de entropía a los fenómenos económicos, corrientes dominantes de la teoría económica todavía recelan de las ventajas y los resultados de ese enfoque metodológico. Esto no ha impedido la aparición ni el desarrollo de disciplinas como la economía cibernética, la programación sistémica, la economía ambiental o la creciente importancia que ha cobrado en la definición de las políticas públicas la estrategia de desarrollo sustentable. Sin embargo, es claro que ese desencuentro ha paralizado el diálogo interdisciplinario, acentuando, si cabe, la necesidad de progreso explicativo más allá de recelos doctrinarios. El presente artículo se inscribe en esa línea de averiguación, aunque su objetivo es más escueto: contribuir a la normativización del estudio de los procesos económicos por medio de una representación entrópica del sector externo de las economías abiertas. Con ese fin elabora un constructo cognitivo basado en la teoría de sistemas bajo dos premisas: ${ }^{1}$ i) una más completa y flexible articulación de los significados de la complejidad, y ii) la superación de los aspectos estériles del debate entre cuantitativistas y cualitativistas. El modelo se compone de siete elementos tradicionales de la sistémica agrupados en dos series: relaciones entrópicas (entropía, homeostasis, selección, restricción y feedback) y componentes básicos (fronteras exteriores y subsistemas). ${ }^{2} \mathrm{Su}$ exposición organiza las secciones del trabajo, precedidas por una sinopsis sobre los principales momentos del estudio entrópico en la economía.

\section{La controversia sobre la aplicación de la entropía}

De acuerdo con el segundo principio de la termodinámica, cuando las formas de moción se detienen en un sistema, las diferencias de potencial eléctrico o químico se

1 El término "constructo cognitivo" es de amplia utilización en la teoría de sistemas; Barton y Haslett (2007) establecen incluso su equivalencia con la totalidad de la doctrina. En el presente artículo el término se emplea en el sentido de modelo conceptual isomórfico de los sistemas complejos.

2 Son tradicionales porque estos elementos no han sufrido cambios mayores en las últimas décadas (Hammond 2002; Atwater et al., 2005; Bawden, 2005). Para su clasificación véase von Bertalanffy (1950:23-29; 1968:80-85); Kremyansky (1960:221-230); Katz y Kahn (1966:86-104); Sommerhoff (1970:147-201), Laszlo (1975) y Blauberg et al. (1977). Desarrollos más reciente, como la autopoiesis (Maturana y Varela 1987) y los sistemas autorreferenciales (Luhman, 1998) se adaptan con dificultad a los procesos económicos o plantean problemas irrelevantes para la presente discusión. Sobre la aplicación de la autopoiesis y las paradojas de la observación, véase también Shkliarevsky (2007). 
igualan y la temperatura deviene uniforme. El sistema alcanza entonces un estado de inercia permanente o supresión de orden interno conocido como entropía máxima. Para evitar ese extremo, el sistema debe exportar entropía e importar negentropía u orden de su entorno. Ello le permite alcanzar mayor complejidad interna y crear nuevos puntos de equilibrio con el macrosistema que lo encierra. Como magnitud, la entropía puede estimarse utilizando la fórmula: $E=K \log D$ donde $\mathrm{K}$ representa la constante de Boltzman $\left(=3.2983 .10^{-24} \mathrm{cal} . /^{\circ} \mathrm{C}\right)$ y D la medida cuantitativa del desorden atómico. Su generalización al conjunto de la física y la química, en seguida a la biología y aun a las ciencias con aplicación social, como la cibernética o la teoría de la información, está a la base de contribuciones cuya importancia científica está fuera de discusión. ${ }^{3}$ En las ciencias económicas, en cambio, las analogías flagrantes y el desarrollo de teorías ambientales (en su mayoría basadas en postulados entrópicos) todavía se encuentran en una etapa incipiente. Esto a pesar de una abundante literatura especializada.

\section{Contribución del enfoque entrópico}

Según la obra seminal de Georgescu-Roegen (1971), el fenómeno de entropía determina las actividades económicas en su conjunto por la simple y suficiente razón de que éstas engloban procesos de degradación de energía. ${ }^{4}$ La premisa es fértil en consecuencias analíticas, una de las más importantes siendo la impugnación de la hegemonía del modelo cíclico por su incapacidad para analizar: el sentido de la reproducción económica, los fenómenos de degradación ecológica y la extinción de recursos naturales. Como opción, Georgescu-Roegen propone un modelo de circulación irreversible y acumulativo más apegado al comportamiento de los flujos reales,

3 La segunda ley de la termodinámica remonta a las contribuciones de Sadi Carnot y Rudolf Claucius en el siglo XIX. Uno de los primeros desarrollos en la física, concretamente en la mecánica cuántica, se debe a von Newmann (1932). Sobre las aplicaciones en la química y los sistemas abiertos, véase Sandler (1989). La versión adaptada a la biología puede consultarse en Schrödinger (1945). Para su empleo en los sistemas vivos, véase Bailey (1990 y 2006). Un trabajo influyente sobre la relación entre entropía y caos es Prigogine y Stengers (1984). Para su aplicación a la cibernética, véase Ashby (1955 y 1963) y Wiener (1961), entre otros. La contribución de la entropía a la teoría de sistemas ha sido reconocida por von Bertalanffy (1968) y Rapoport (1986).

4 Para el desarrollo de las tesis de Georgescu-Roegen, véanse los ensayos coordinados por Tang et al. (1976). Khalil (1990) ha elaborado una crítica radical del paradigma del economista rumano apelando a una distinción de las definiciones de Clausius y Kelvin de la entropía. Una impugnación interesante de las premisas de Khalil pertenece a Lozada (1991). La reposición actual de Georgescu-Roegen ha sido realizada por Daly (1996). 
donde una parte de los insumos no son renovables. En la prolongación de este argumento, Daly (1996) opone el desarrollo sustentable al crecimiento económico simple, y defiende la formación de una "conciencia entrópica" que sirva al valor mediante la reducción del deterioro del ambiente. Rifkin (1980) sustancia otro tema importante para esta literatura: la rivalidad entre los enfoques microscópico y macroscópico. En oposición a Boltzman, en cuya fórmula está la base de los estudios técnico-estadísticos, el autor afirma que la entropía se capta mejor cuando se consideran los elementos difusos y homogéneos que produce el sistema y no los aspectos microscópicos del status quo ante. La entropía representaría en ese contexto "una medida de la cantidad de energía que ya no puede transformarse en trabajo" (1980:35).

Macrakis (1997) también se ocupa de la controversia micro-macroscópica, la cual considera afectada por la circularidad de sus argumentos: "Todos los constructos teóricos se originan en la imagen de los fenómenos macroscópicos, mientras que todos los fenómenos microscópicos sólo pueden ser inferidos pero nunca directamente observados a través de los efectos macroscópicos" (1997:105). Boulding (1976) insiste en la defensa de la visión cualitativa de la entropía y la recomienda como una suerte de guía o síntoma de la robustez organizativa del sistema. Con ese fin la entropía iguala la ley de difusión, i.e. cuando las concentraciones de elementos tienden a hacerse difusas como producto de su actividad. Más cercano a nuestro tema, Pulliainen (1970) apela al isomorfismo entre comercio internacional y los sistemas de comunicación para estimar la entropía del intercambio entre bloques comerciales. Con ese fin adopta el instrumental matemático de la teoría de la información de Shannon y Weaver (1963), si bien con ello cae en el reduccionismo estadístico y el consecuente abandono de los aspectos cualitativos del fenómeno. ${ }^{5}$

\section{Discrepancias}

A pesar del número y la calidad de estos trabajos, no son escasas las tomas de posición contrarias a la plena generalización de la entropía. Espaciados por más de medio siglo, Jeans (1933), Wasylenko y Erickson (1978) y Khalil (1990) afirman que los fenómenos sociales, incluyendo a los económicos, no obedecen la ley de la termodinámica. Las conclusiones de Altvater (1994), análogas aunque inscritas en un enfoque marxista, sostienen que la circularidad y el tiempo entrópico no son sustituibles

5 Para otros trabajos que emplean los fundamentos de la entropía en las ciencias económicas, véase Murphy (1965); Theil (1967); Hackbart y Anderson (1975); Hsieh y Ye (1991); Fraser (2000), entre otros. 


\section{4}

GERMÁN A. DE LA REZA

porque la forma de producción capitalista los contrapone. Jaynes (1991), finalmente, observa que una adecuada aplicación de la entropía necesita primeramente conocer cuáles variables macroeconómicas deben entrar en el análisis y cuáles hipótesis espaciales microeconómicas determinan la multiplicidad de factores. Vista la dificultad en lograr ambas operaciones, el autor advierte: "Una real implementación de esta analogía termodinámica todavía pertenece a un futuro remoto" (1991:5).

Llegados aquí, es necesario interrogarnos sobre la posibilidad de zanjar el debate sobre la validez de la entropía en la economía. La tentación de operar con nuevas cuantificaciones o fusiones teóricas no debe oscurecer el hecho de que en sus términos actuales la cuestión es básicamente insoluble. Desde el punto de vista epistemológico estamos ante la rivalidad de paradigmas científicos cuyo desenlace depende no sólo de la superioridad racional, sino también de leyes sociológicas (Kuhn, 1970). La perspectiva ontológica arroja una respuesta complementaria: las teorías económicas y entrópicas se refieren a fenómenos que se fundan en la realidad de manera específica y sólo en última instancia son isomórficos. Esa instancia, cabe precisar, tiene en un extremo al reduccionismo simplificador de la transdisciplina, y en el otro la ausencia de parámetros interdisciplinarios universalmente aceptados.

\section{Una representación de la complejidad}

Una opción frente a los aspectos estériles de este debate consiste en reconstruir el objeto de estudio al interior de una metodología que fundamente y norme el transporte de técnicas y conceptos entre disciplinas. La construcción de ese modelo con base en la teoría de sistemas debe considerar dos pasos previos. En primer lugar, la selección de un lenguaje que no sea económico ni tampoco entrópico, sino que articule las principales relaciones de un sistema complejo con el entorno. En segundo lugar, la especificación del modelo en función de variables endógenas y exógenas por igual. Este requerimiento resulta de la equivalencia analítica entre el sector externo $\left(\mathrm{S}_{\mathrm{E}}\right)$ de una economía abierta $\left(\mathrm{E}_{\mathrm{A}}\right)$ y el subsistema de un sistema abierto. ${ }^{6}$ Los principales elementos del modelo se resumen en la figura 1.

6 El concepto de "sistema abierto" es enunciado por von Bertalanffy (1950) como parte fundamental de su propuesta de generalización de la sistémica biológica. Como se verá más adelante, el sistema abierto designa por igual a un modelo interpretativo y al conjunto de intercambios con el entorno y por su mediación, la serie de modificaciones (en componentes y relaciones) que éstos procuran al sistema" (De la Reza 2001:78). Para una discusión reciente sobre las ventajas metodológicas del concepto, véase Barton y Haslett (2007). 
Figura 1

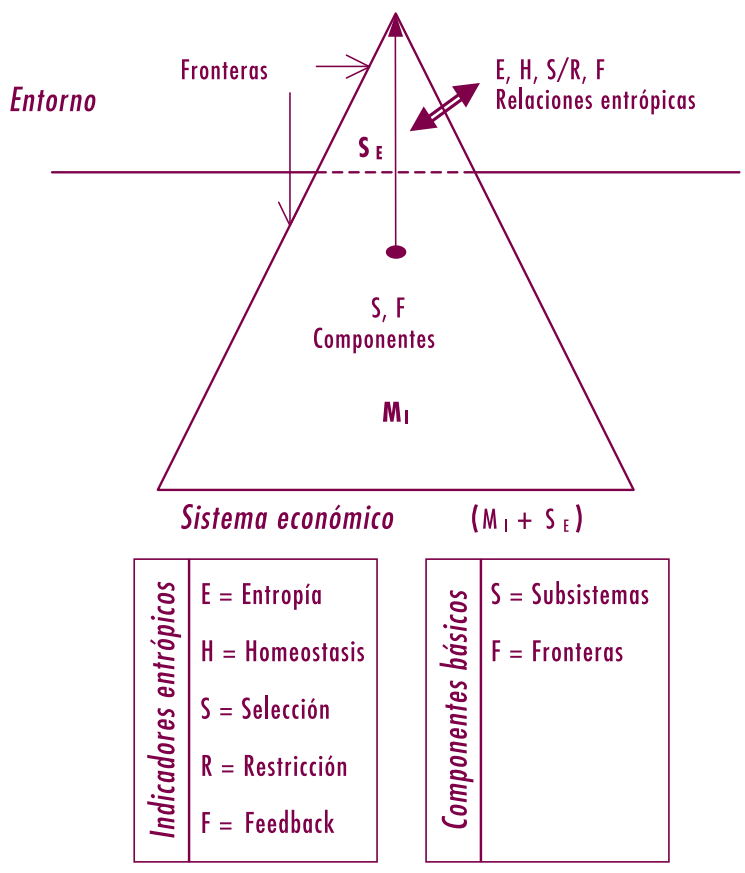

$\mathrm{E}_{\mathrm{A}}$ representa la suma de los subsistemas $\mathrm{S}_{\mathrm{E}} \mathrm{y}$ mercado interno $\left(\mathrm{M}_{\mathrm{I}}\right)$. $\mathrm{S}_{\mathrm{E}}$ interacciona con el entorno mediante cinco tipos de relación: entropía, homeostasis, selección, restricción y feedback. En cada relación existe una polaridad entre dos extremos ideales cuyo punto de equilibrio corresponde a la situación de estabilidad y expansión. Los componentes básicos del constructo son estructura interna (subsistemas) y diferenciación con el entorno (fronteras). Para evitar las imprecisiones del enfoque holista, las relaciones se definen apelando a criterios cualitativos y a variables estadísticas. Por ese medio se pretende que los agregados y la deducción, esenciales para el enfoque macroeconómico, retroalimenten el análisis y las operaciones inductivas del enfoque microeconómico. Ese vínculo convierte ambos niveles en los términos inseparables de una misma relación cognitiva (Holton 1998:111-113).

\section{Entropía}

La entropía parte de la premisa según la cual toda $\mathrm{E}_{\mathrm{A}}$ importa alguna forma de energía de su entorno $\left(\mathrm{E}_{\mathrm{N}}\right)$ y la devuelve después de un proceso de transformación. El término 


\section{6}

GERMÁN A. DE LA REZA

energía designa al conjunto de sucesos, bienes de consumo, materias primas, bienes de capital, tecnología, servicios, capitales, mano de obra, etcétera, intercambiados externamente y que contribuyen a la reproducción económica. Cuando $S_{E}$ pierde importancia y el sistema tiende a la autarquía, los flujos de entrada decrecen su valor para la reproducción del sistema pero acrecientan su efecto sobre los desequilibrios y las inadaptaciones al entorno. Esto puede verse como una polaridad entre desorden y rigidez, donde la cercanía a ambos extremos impide al sistema realizar los ajustes necesarios para una eficiente inserción en la economía internacional (Wiener 1961:11).

En principio, las variables que definen la relación de $\mathrm{E}_{\mathrm{A}}$ con el entorno son numerosas en razón de la complejidad del fenómeno y de las propias aspiraciones del contructo. ${ }^{7}$ En $\mathrm{S}_{\mathrm{E}}$ las variables abarcan seis áreas: i) comercio de bienes y servicios; ii) flujos financieros; iii) energía y ambiente; iv) salud; v) educación, y vi) información. En el comercio se consideran: la magnitud absoluta del intercambio, la tasa de crecimiento y la participación en el Producto Interno Bruto (PIB) de las importaciones y las exportaciones (grado de apertura de $\mathrm{E}_{\mathrm{A}}$ ); la estructura (o proporción de productos primarios, manufacturados y de alta tecnología), y la relación de intercambio (relación entre el índice de precios de importación y exportación a partir de un determinado año). En los flujos financieros importan la estructura de las inversiones (proporción de la nueva inversión extranjera directa sobre los créditos intrafirma y la inversión de cartera) y el nivel de endeudamiento (o pago de intereses como porcentaje del total de las exportaciones). En energía y ambiente se investigan las dotaciones de agua, la dependencia frente al petróleo (o consumo de combustibles tradicionales como porcentaje de las necesidades industriales totales) y las emisiones de dióxido de carbono per capita y como porcentaje del total mundial. La salud y educación se estudian apelando a indicadores tradicionales, como esperanza de vida, control de enfermedades y nivel de alfabetización. La información, finalmente, comprende: transferencia de tecnología, asistencia técnica, acceso a conocimiento especializado, entre otras variables. ${ }^{8}$

7 La amplitud que caracteriza a los modelos sistémicos no ha sido ajena al carácter heurístico y experimentalmente difuso de sus estudios. Puede conjurarse ese riesgo, al menos en parte, apelando a variables más precisas, criterio que seguimos en el presente artículo.

8 La mayoría de esas variables está disponible en los anuarios de Naciones Unidas sobre el índice de desarrollo humano, además éstas se elaboran con base en metodologías verificadas (PNUD 2007). El grupo de variables que utilizamos ha sido estudiado por World Watch Institute (2005), en particular: la excesiva dependencia frente al petróleo, el difícil acceso a las fuentes de agua potable; necesidades de alimentación, las enfermedades infecciosas y el desempleo en jóvenes. 


\section{Homeostasis}

La homeostasis designa, a su vez, a las fuerzas de reproducción que no afectan a los elementos esenciales del sistema, sino que los multiplican incorporando unidades de tipo similar (De la Reza 2001:163). El proceso no es de simple renovación, ya que la expansión homeostática reemplaza las funciones difusas por otras más definidas, teniendo por objetivo final el que "cualquier organización individual realice una sola función específica, o cuanto mucho, un pequeño grupo de funciones estrechamente relacionadas" (Deutsh 1965:212). Para asegurar su viabilidad, el proceso se asocia a una creciente jerarquía, sentando las bases de la configuración de $\mathrm{S}_{\mathrm{E}}$ como un sistema complejo. Por definición, la homeostasis difiere y claramente se contrapone a los cambios que desarticulan al sistema como un todo.

El caso de la apertura comercial y la consiguiente necesidad de adaptación a una nueva estructura de precios permiten diferenciar la homeostasis dinámica y el desorden. Tomemos el caso de la revolución industrial inglesa de principios del siglo XIX y su doble efecto internacional. En los países vecinos y mejor preparados favoreció la adopción de modelos de industrialización parecidos o idénticos al británico, incluyendo la compra de maquinaria, la asistencia técnica y el desarrollo gerencial (Brulant, 1989). ${ }^{9}$ En Hispanoamérica, por el contrario, la abierta exposición a la competencia y la ausencia de políticas industriales entre 1820 y 1830 condujo a la masiva importación de textiles ingleses y con ello a la desaparición de la artesanía textil o de obrajes (Bulmer-Thomas 1994:42-43)..$^{10}$ En un caso asistimos a un salto cualitativo estimulado por el entorno y que aprovecha parámetros internos (homeostasis) y en el otro a una aguda crisis del sistema económico.

El indicador global de la homeostasis es el crecimiento económico, el cual se desagrega en variables más precisas como la tasa de crecimiento anual del PIB

9 La tesis difusionista de la industrialización ha sido defendida por Pollard (1981). Una posición más aislada, que acentúa la especificidad de los procesos manufactureros, tiene entre sus exégetas a Kemp (1978). El estudio de Brulant (1989) aporta evidencias de que la difusión de la tecnología de la industria textil inglesa en Noruega fue promovida por los propios ingleses en la forma de paquetes que incluían la asistencia técnica.

10 En la década de los veinte del siglo XIX, la compra de textiles ingleses se convierte en el principal rubro de importación hispanoamericana, estimulada por sus mejores precios y la ausencia de adecuadas políticas gubernamentales en los países de la región, endeudados y con una base industrial arruinada por la guerra de la Independencia. La situación no está exenta de excepciones, como el caso de México, donde una férrea política arancelaria, al origen de las revueltas antigubernamentales de 1841 y 1851 , permite la supervivencia del sector (Keremitsis 1973:31 y s.). 


\section{8}

GERMÁN A. DE LA REZA

(expansión homeostática), el valor más alto durante un determinado periodo (las magnitudes de la accidentalidad) y la estructura del producto interno. Esta última información permite comprender la intensidad en los cambios y su efecto sobre los parámetros constantes del sistema. Se entiende que si los valores fluctúan de manera acentuada, el intercambio con el entorno puede alterar al sistema en sus aspectos esenciales.

\section{Selección y restricción}

La selección y la restricción comportan objetivos específicos, aunque en ocasiones sea difícil diferenciarlos. El primer caso consiste en operaciones de diferenciación de determinadas funciones con respecto a algún sistema o subsistema, e involucra en algún momento (y a diversos grados) una fuerza interna de rechazo. El resultado puede verse como una multiplicidad de vectores que impulsan la adaptación o la modificación del sistema. En circunstancias extremas, como la apertura comercial indiscriminada, la ausencia de mecanismos selectivos provoca una avalancha de elementos externos que hacen pesar una amenaza de desintegración sobre el sistema. En un sentido más amplio, la selección corresponde al grado de especialización del sistema dentro de un patrón de actividades corregidas por numerosas posiciones de retroalimentación. Obsérvese que si $\mathrm{E}_{\mathrm{A}}$ corresponde a una economía pequeña, la especialización productiva resulta más una condición de origen que una elección. Paradójicamente, esa situación incrementa la vulnerabilidad del sistema en la medida que reduce sus opciones frente a sustanciales cambios en la demanda externa.

La restricción, por su parte, engloba a un conjunto de opciones propias que limitan a las posibilidades foráneas y viceversa (Ashby 1955). Visto desde fuera, todo entorno relevante para el sistema (o todo sistema respecto de sus subsistemas) genera procesos de restricción en la forma de correctores de objetivos, opciones o acciones del sistema (o subsistema). El desenvolvimiento de esas restricciones es contrarrestado por la capacidad de respuesta interna del sistema (o subsistema), la cual se manifiesta por medio de diversos instrumentos de reacción y adaptación (Buckley 1966:63). En su conjunto, los instrumentos componen una suerte de fuerza general restauradora que crece y se diversifica a medida que el sistema aumenta su propia estructuración. Se entiende que la reacción del sistema a las restricciones externas también incide en la organización del entorno, por mucho que dicha reacción se concentre en un determinado suceso (Deutsh, 1974). Sus instrumentos se dividen en dos categorías: controles fijos y contingentes, concretamente, barreras arancelarias y no arancelarias. 
Las primeras no presentan dificultad para el estudio por tratarse de impuestos establecidos oficialmente, aunque las reducciones arancelarias pactadas en las rondas del Acuerdo General sobre Aranceles Aduaneros y Comercio (GATT), la Organización Mundial del Comercio (OMC) y en los tratados de libre comercio han hecho de ellas un indicador poco significativo. Las segundas, por el contrario, están en continuo desarrollo: medidas antidumping; derechos compensatorios; excesivos controles aduaneros, fitosanitarios y ambientales; subvenciones a las industrias sensibles, compras gubernamentales y otros. Obsérvese que el carácter contingente de estas barreras no facilita la anticipación de sus efectos, aunque se asume que generalmente son anticompetitivos. Desde esa lógica, el empleo indiscriminado de los instrumentos de restricción conduce el sistema al cierre y a una creciente inadaptación frente al entorno. Su total ausencia, en cambio, provocaría la desintegración de la entidad.

\section{Feedback}

Para una representación eficiente de las discrepancias entre entradas y salidas de un sistema es necesario apelar al feedback o retroalimentación. ${ }^{11}$ Este tipo de causación suele explicarse apelando al termostato. La temperatura de una habitación cualquiera representa el output del termostato; si éste es menor al input de referencia (la temperatura objetivo del termostato), el artefacto entra en funcionamiento para aumentar el output hasta el nivel del input. Cuando esto ocurre, el calefactor se detiene, manteniendo el nivel deseado de temperatura. En términos formales, el mecanismo puede expresarse de la siguiente manera:

$$
\begin{aligned}
& \text { (1) } y=\mathrm{A}(x+\mathrm{B} y) \\
& \text { (2) } y=\mathrm{A} x /(1-\mathrm{AB}) \\
& \mathrm{G}=y / x=\mathrm{A} / 1-\mathrm{AB}
\end{aligned}
$$

donde $\mathrm{G}$ es la ganancia del sistema, A el amplificador, $y$ el output, $x$ el input y B la red de retroalimentación. Desde un enfoque cualitativo, el feedback representa una causalidad multilateral recíproca con doble acción: i) negativa, cuando amplifica las diferencias entre el valor del objetivo y el valor de la salida, y ii) positiva, cuando la causalidad disminuye dichas diferencias. Se entiende que la diferencia entre una y

11 La importancia del feedback para la comprensión de los sistemas sociales ha sido defendida por Burmeister (1978:68); Maruyama 1963:164-179), Deutsh (1963) y Buckley (1966), entre otros. 


\section{0}

GERMÁN A. DE LA REZA

otra (la cual no siempre es idéntica desde otros puntos de vista) establece la magnitud de la restauración del equilibrio (Ashby 1963). ${ }^{12}$ Por otro lado, dicha diferencia lleva implícito un sentido y éste puede ser multifinal, i.e. cuando a los estados iniciales similares les corresponde estados finales diferentes (Buckley, 1966).

Las discrepancias entre el output y el input de $\mathrm{E}_{\mathrm{A}}$ se reflejan en la balanza de pagos, el registro generalmente anual que mantiene informado al sistema sobre los pagos al exterior (débito anotado con signo negativo) y los ingresos provenientes de la venta de productos de exportación (crédito anotado con signo positivo). En caso de déficit crónico, $\mathrm{E}_{\mathrm{A}}$ debe incrementar su competitividad o buscar la aplicación de políticas restrictivas, como subvenciones, políticas fiscales, compras gubernamentales, tipo de cambio y barreras al comercio. El grado de utilización de esas políticas depende de las industrias que se busca proteger, la intensidad del desequilibrio externo y los compromisos internacionales contraídos sobre todo en materia comercial y financiera. Aquí estamos nuevamente ante un continuo de acciones de restauración del equilibrio y promoción del desarrollo. Asimismo, ante una dialéctica que asocia y hasta cierto punto modela las operaciones de síntesis y análisis (Ritchey 1996:2).

\section{Componentes básicos del sistema}

En un sentido pleno, las relaciones entrópicas también son componentes del sistema, aunque su manifestación como fuerzas y estados dinámicos los distingue de los elementos objetuales del modelo. Dos componentes son imprescindibles para la cabal definición de nuestro constructo cognitivo: los subsistemas y las fronteras externas.

\section{Subsistemas}

La estructura y la función de un subsistema dependen del grado de complejidad del sistema al que pertenece. Desde un punto de vista ideal, se tiene tres casos distintos: i) subsistemas de "simplicidades organizadas" o sistemas menos complejos, en los cuales se observa una tendencia a la rigidez y a un menor margen de maniobra adaptativo; ii) subsistemas de "complejidades organizadas", los cuales aglutinan mayor información, orden y especialización (Rapoport y Horvath 1959:98); y iii) subsistemas de entidades difusas y multiestables, caracterizados por un posicionamiento irregular, menor número de parámetros esenciales y una inestabilidad permanente o prolongada. Nótese que esta tipología sólo se refiere a estados o condiciones generales. En los

12 Véase también Kim (1975:207-208), y Bigelow et al. (1943). 
sistemas reales los parámetros más simples coexisten con otros altamente estructurados y su desenvolvimiento combina diferentes grados de organización.

El término subsistema tiene el doble carácter de objeto y modelo conceptual. Para explicar el segundo sentido tomemos el caso de la rama industrial textil de un país cualquiera. Desde una de sus perspectivas, la rama puede verse como un sistema compuesto de distintas cadenas productivas o industrias en la posición de subsistemas. Desde otra perspectiva, esa rama constituye el subsistema de la producción manufacturera. En una tercera, representa la unidad económica de una región cualquiera (subsistema) de un país. En este trabajo la definición de subsistema considera las particularidades de $S_{E}$, sus tres subsubsistemas importantes (comercio, finanzas y producción) y un número determinado de elementos o unidades (empresas) que realizan funciones y ocupan posiciones jerárquicas específicas.

\section{Fronteras del sistema}

El término "fronteras del sistema" no implica delimitación espacial, sino identidad o pertenencia de los subsistemas, subsubsistemas, elementos o unidades menores a una determinada entidad. En ocasiones un espacio coincide con la esfera de implantación de los elementos del sistema, aunque incluso en ese caso la dimensión territorial aparece subordinada a la pertenencia de los objetos. En toda $\mathrm{E}_{\mathrm{A}}$ la definición de las fronteras raras veces es exhaustiva, ya que sus elementos pueden tener una posición intermedia en dos o más sistemas, o tornarse cada vez más difusos conforme se aproximan al límite externo del sistema. La doble pertenencia puede asumir las más diversas formas: zonas libres, empresas transnacionales, inversión extranjera, trabajadores emigrantes, clusters, etcétera. Los asalariados españoles, mexicanos o haitianos que laboraban en la década de los sesenta del siglo pasado en el sur vitícola de Francia, en diversas localidades de Estados Unidos y en la parte occidente de la República Dominicana, podían ser sujetos legales en ambos países durante el periodo de migración. Las remesas completaban los efectos de esa doble pertenencia.

Como en el caso de las relaciones entrópicas, la pertenencia al sistema actúa entre dos extremos: la independencia de los elementos y una estrecha unión. Nótese que el punto medio de esas polaridades no es estático; la evolución reciente de los sistemas económicos muestra una tendencia a la subsistemización jerárquica de economías enteras. La reestructuración de países de América Latina a partir de la década de los ochenta estimuló la reorganización espacial de la producción; la interpenetración de las industrias transnacionales; la relativa continuidad de los mercados de capitales internacionales; la homologación de los bienes de consumo a escala planetaria, y el 


\section{2}

GERMÁN A. DE LA REZA

movimiento transfronterizo de la fuerza de trabajo (Mittelman 1994:427). Esos procesos se asociaron a su vez a la subsistemización de las economías latinoamericanas bajo la lógica de una nueva y más compleja división internacional del trabajo.

\section{Análisis mutivariable sin determinación teórica causal}

Una principal ventaja del constructo definido en estas páginas es que permite asociar los niveles cualitativo y técnico-estadístico del análisis. En el primero se atienden las especificidades, los agregados y las magnitudes de los cinco tipos de relación y con esa información se elabora un diagnóstico general de la entropía del sistema. En el segundo nivel se opera con las variables de cada relación, apelando para su interpretación a las distintas técnicas de la estadística y la econometría. Para establecer el tipo de evolución económica, por ejemplo, se recurre a la tasa de crecimiento; el valor de los accidentes se puede conocer mediante la dispersión (distancia frente a la media) o la desviación estándar; para medir la dependencia mutua de un grupo de variables se apela al coeficiente de correlación o la regresión múltiple (efecto de las variables exógenas sobre la variable endógena o dependiente); los grados de endogenidad de las variables, a los vectores autorregresivos (VAR), etcétera.

El VAR es útil cuando estamos ante el caso (frecuente) de variables simultáneas cuyo vínculo causal se desconoce y que en consecuencia deben tratarse sobre una base de igualdad, sin distinciones a priori (Sims, 1980). ${ }^{13}$ Para construir el modelo VAR de la entropía del $\mathrm{S}_{\mathrm{E}}$, se requiere primeramente especificar las variables. Para ilustrar el procedimiento tomemos la evolución del comercio internacional (importaciones más exportaciones, IE) y la tasa arancelaria aplicada a las importaciones (TA). En cada ecuación hay cinco valores rezagados (variables con efecto diferido) de IE y cinco valores rezagados de TA como regresores, y los $\mu$ son "impulsos" o términos de error estocásticos. El modelo resultante es:

$$
\begin{aligned}
& \text { (1) } \mathrm{IE}_{t}=\alpha+\sum_{j=1}^{5} \beta_{j} \mathrm{IE}_{t-j}+\sum_{j=1}^{5} \gamma_{j} \mathrm{TA}_{t-j}+\mu_{1 t} \\
& \text { (2) } \mathrm{TA}_{t}=\alpha^{\prime}+\sum_{j=1}^{5} \theta_{j} \mathrm{IE}_{t-j}+\sum_{j=1}^{5} \lambda_{j} \mathrm{TA}_{t-j}+\mu_{2 t}
\end{aligned}
$$

Una vez definido el VAR, introducimos los valores de TA e IE y el modelo corre de manera sencilla. A pesar de su sofisticación, este tipo de modelo no es el único

13 Para una explicación sucinta de los modelos VAR véase Gujarati (1997: 729-734). 
pertinente. Para medir los efectos causales dentro del sistema, se puede apelar a: i) la prueba de exogenidad en bloque, la cual establece el rango de las variables más independientes o más endógenas; y ii) la prueba de Granger, uno de los antecedentes de VAR, la cual mide el valor causal ("en el sentido de Granger") o estadísticamente significativo de la variable. Dicho esto, a pesar de que gran parte de las relaciones puede estudiarse a partir de series estadísticas, la comprensión de las interacciones y las magnitudes dependen del estudio cualitativo. Los niveles sintético y analítico aparecen así como partes interconectadas de un mismo proceso de investigación.

\section{Reflexiones finales}

Diversos autores que emplean la entropía para la comprensión de los procesos económicos han mostrado las limitaciones del enfoque cíclico tradicional, así como la necesidad de completarlo con un modelo feedback multifinal que capte las discrepancias entre entradas y salidas. Sin embargo, la localizada influencia de esas contribuciones y el estancamiento del debate interdisciplinario hacen necesaria la exploración de espacios metodológicos alternativos. El constructo cognitivo que hemos presentado en estas páginas tiene por objetivo llenar ese vacío mediante el afianzamiento metodológico. Para lograr ese objetivo, el modelo se dotó de cuatro refuerzos:

i) Explicación del objeto de estudio dentro de un contexto metodológico normativo y adaptado al transporte interdisciplinario;

ii) Transformación del vínculo entre micro y macro niveles en un proceso continuo entre dos extremos;

iii) Análisis de las relaciones con el entorno a partir de un número amplio de variables, definiendo en cada caso los equilibrios óptimos para el sistema; y

iv) Definición de los parámetros del estudio aplicado, énfasis necesario para la normativización del estudio de la entropía en la economía.

Esas características no eliminan los inconvenientes de trasladar un objeto de estudio fuera de las teorías en las que ha acumulado gran cantidad de conocimiento. Entre los riesgos a tener en cuenta resalta la pérdida de información resultante de las operaciones de transporte. Para evitarla, es necesario que el constructo establezca parámetros relativamente sencillos para el transporte de técnicas y conceptos, y estimule el empleo de técnicas interdisciplinarias. En los hechos, la respuesta a esos desafíos constituye la clave de la aportación del estudio entrópico a la teoría del desarrollo económico sustentable. 


\section{Bibliografía}

Altvater, E., "Ecological and Economic Modalities of Time and Space", en M. O'Connor (ed.) Is Capitalism Sustainable?, Nueva York, Guilford Press, 1994, pp. 37-52.

Ashby, W. R., "Self-Regulation and Requisite Variety", Introduction to Cybernetics, John Wiley, Chichester, 1955, pp. 202-218. Reimpreso en F. E. Emery (ed.) Systems Thinking, Harmondsworth, Penguin Books, 1969, pp. 105-124.

, An Introduction to Cybernetics, Chichester, John Wiley, 1963.

Atwater, B.J., V. Kannan, y A.A. Stephens, "Teaching Systemic Thinking: Educating the Next Generation of Business Leaders", Systems Thinker, núm. 16, 2005, pp. 1-5.

Bailey, K. D., Social Entropy Theory, Albany, State University of New York Press, 1990.

,"Living Systems Theory and Social

Entropy Theory", Systems Research and Behavioral Sciences, núm. 23, 2006, pp. 291-300.

Barton, J. y T. Haslett, "Analysis, Synthesis, Systems Thinking and the Scientific Method: Rediscovering the Importance of Open Systems", Systems Research and Behavioral Sciences, núm. 24, 2007, pp. 143-155.

Bawden, R., "Systems Development at Hawkesbury: Some Personal Lessons from Experience", Systems Research and Behavioral Sciences, núm. 22, 2005, pp. 151-164.

Bigelow, J., A. Rosenblueth y N. Wiener, "Behavior, Purpose, and Teleology", Philosophy of Science, núm. 10, 1943, pp. 18-24. Reimpreso en W. Buckley (ed.) Modern Systems Research for the Behavioral Scientist, Chicago, Aldine Publishing Co., 1972, pp. 221-225.

Blauberg, I. V., V. N. Sadovsky y E. G. Yudin, Systems Theory: Philosophical and Methodological Problems, Moscú, Progress Publishers, 1977.

Boulding, K. E., "The Great Laws of Change" Evolution", A. Tang et al. (eds.) Welfare, and Time in Economics: Essays in honor of Nicholas Georgescu-Roegen, Lexington, Lexington Books, 1976, pp. 3-21.

Brulant, K., British Technology and European Industrialization: The Norwegian Textile Industry in the Mid Nineteenth Century, Nueva York, Cambridge University Press, 1989.
Buckley, W., Sociology and Modern Systems Theory, Englewood Cliffs, Prentice-Hall, Nueva Jersey, 1966. Trad. española, $L a$ sociología y la teoría moderna de los sistemas, Editorial Amorrortu, Buenos Aires, 1973.

Bulmer-Thomas, V., The Economic History of Latin America since Independence, Nueva York, Cambridge University Press, 1994.

Burmeister, E., "Expectations and Dynamic Stability in Economic Models", Recent Developments in Variable Structure Systems, Economics and Biology, Berlin, SpringerVerlag, 1978, pp. 68-95.

Daly, H. E., Beyond Growth: the Economics of Sustainable Development, Boston, Beacon Press, 1996.

De la Reza, G. A., Teoría de sistemas. Reconstrucción de un paradigma, México, Porrúa, 2001.

Deutsh, K. W., The Nerves of Goverment, Nueva York, The Free Press, 1963.

"Supranational Organizations in the 1960's", Journal of Common Market Studies, vol. 16, 1965, pp. 212-218.

"On the Interaction of Ecological and Political Sciences: Some Potential Contributions of the Social Sciences to the Study of Man and His Environment", Social Science Information, 13 de junio de 1974, pp. 5-15.

Fraser, I., "An application of maximum entropy estimation: the demand for meat in the United Kington", Applied Economics, vol. 32, 2000, pp. 45-59.

Georgescu-Roegen, N., The Entropy Law and the Economic Process, Cambridge, Harvard University Press, 1971.

Gujarati, D. N., Econometría, Bogotá, McGraw-Hill, 1997.

Hackbart, M. W. y D. A. Anderson, "On Measuring Economic Diversification", Land Economics, vol. 51, 1975, pp. 374-378.

Hammond, D., "Exploring the Genealogy of Systems Thinking", Systems Research and Behavioral Sciences, núm. 19, 2002, pp. 429-439.

Holton, G., The Scientific Imagination, Harvard University Press, Cambridge, 1988.

Hsieh, C. Y. y M. H. Ye, Economics, Philosophy, and Physics, Nueva York, M. E. Sharpe, Inc., 1991. 
Jaynes, E. T., "How should we use entropy in economics?", 1991 http://bayes.wustl.edu/ etj/articles/ entropy.in.economics.pdf.

Jeans, J. H., The New Background of Science, Cambridge, Cambridge University Press, 1933.

Katz, D. y R. L. Kahn, "Common Characteristics of Open Systems", The Social Psychology of Organizations, Wiley, 1966, pp. 14-29. Reimpreso en F. E. Emery (ed.) Systems Thinking, Harmondsworth, Penguin Books, 1970, pp. 86-104.

Kemp, T., Historical Patterns of Industrialization, Londres, Longman, 1978.

Keremitsis, D., La industria textil mexicana en el siglo XIX, Colección Septentas, México, SEP, 1973.

Khalil, E. L., "Entropy Law and Exhaustion of Natural Resources: Is Nicholas GeorgescuRoegen's Paradigm Defensible", Ecological Economics, vol. 2, 1990, pp. 163-178.

Kim, J. Y., "Feedback in Social Sciences: Toward a Reconceptualization of Morphogeneses", J. Y. Kim y B. D. Ruben (eds.) General Systems Theory and Human Communication, Nueva Jersey, Hayden Book Company, 1975, pp. 207-221.

Kuhn, T. S., The Structure of Scientific Revolutions, Foundations of The Unity of Science, vol. II, Chicago, The University of Chicago Press, 1970.

Kremyansky, V. I., "Certain Peculiarities of Organisms as a 'System' from the Point of View of Physics, Cybernetics, and Biology", Year Book of the Society for General Systems Research, vol. V, 1960, pp. 221-230.

Laszlo, E., "Basic Constructs of Systems Philosophy" B. D. Ruben y J. Y. Kim (eds.) General Systems Theory and Human Communication, Nueva Jersey, Hayden Book Company, 1975, pp. 66-77.

Lozada, G. A., "A defense of Nicholas Georgescu-Roegen's Paradigm", Ecological Economics, vol. 3, 1991, pp. 157-160.

Luhmann, N., Sistemas sociales. Lineamientos para una teoría general, Barcelona, Anthopos, Universidad Iberoamericana, CEJA, 1998.

Macrakis, M. S., Scarcity's Way: the Origins of Capital, Dordrecht, Kluwer Academic Publishers, 1997.

Maruyama, M., "The Second Cybernetics, Deviation-Amplifying Mutual Causal Process", American Scientist, núm. 51, 1963 , pp. 164-179. Reimpreso en Year Book of the Society for General Systems Research, vol. VIII, 1983, pp. 233-241.

Maturana, H. R. y F. J. Varela, El árbol del conocimiento. Las bases biológicas del entendimiento humano, Santiago, Editorial Universitaria, 1987.

Mittelman, J. H., "The Globalization Challenge: Surviving at the Margins", Third World Quaterly, vol. 14, núm. 3, 1994, pp. 155-164.

Murphy, R. E., Adaptative Processes in Economic Systems, Nueva York, Academic Press, 1965.

PNUD, Informe sobre desarrollo humano 2006, Ginebra, PNUD, 2007.

Pollard, S., Peaceful Conquest. The Industrialization of Europe 1760-1970, Oxford, Oxford University Press, 1981.

Prigogine, I. y E. I. Stengers, Order out of Chaos, Nueva York, Bantam Books, 1984.

Pulliainen, K., "Entropy Measures for International Trade", The Swedish Journal of Economics, vol. 72, núm. 1, 1970, pp. 40-73.

Rapoport, A., General System Theory, Essential Concepts \& Applications, Cambridge, Abacus Press, Tunbridge Wells, Kent, 1986.

y W.J. Horvath, "Thoughts on Organization Theory", Year Book of the Society of General Systems Research, vol. IV, 1959, pp. 17-32.

Rifkin, J., Entropy: a New World View, Nueva York, Viking Press, 1980.

Ritchey, T., Analisis and Synthesis. On Scientific Method, 1996 http://www.swemorph. com/pdf/ anaeng-r.pdf.

Sandler, S. I., Chemical and Engineering Thermodynamics, Nueva York, John Wiley \& Sons, 1989

Schrödinger, E., "Order, Disorder, and Entropy", What is Life, Cambridge, Cambridge University Press, 1945. Reimpreso en W. Buckley (ed.) Modern Systems Research for the Behavioral Scientist, Chicago, Aldine Publishing Company, 1968, pp. 143-160.

Shannon, C. E. y W. Weaver, The Mathematical Theory of Communication, Urbana, 1963.

Shkliarevsky, G., "The Paradox of Observing, Autopoiesis, and the Future of Social Sciences", Systems Research and Behavioral Sciences, 2007, núm. 24, pp. 323-332.

Sims, C. A., "Macroeconomics and Reality", Econometrica, vol. 48, 1980, pp. 1-48. 


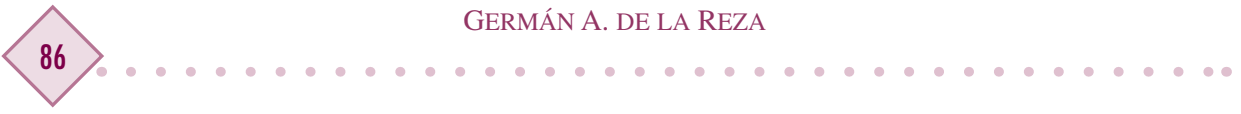

Sommerhoff, G., "The Abstract Characteristics of Living Systems", F. E. Emery (ed.) Systems Thinking, Harmondsworth, Penguin Books, 1970, pp. 147-202.

Tang, A. et al. (eds.) Welfare, and Time in Economics: Essays in honor of Nicholas Georgescu-Roegen, Lexington, Lexington Books, 1976.

Theil, H., Economics and Information Theory, Amsterdam, North Holland Publishing Company, 1967.

Von Bertalanffy, L., "The Theory of Open Systems in Physics and Biology", Science, vol. III, 1950, pp. 23-29. Reimpreso en F. E. Emery (ed.), Systems Thinking, Harmondsworth, Penguin Books, 1969, pp. 70-85.
, General Systems Theory. Foundations, Development, Applications, Georges Braziller, Nueva York, 1968. Trad. española: Teoría general de los sistemas, México, Fondo de Cultura Económica, 1976.

Von Newmann, J., Mathematische Grundlagen der Quantenmechanik, Springer-Verlag, Berlín, 1932 (1996).

Wasylenko, M. J. y R. A. Ericsson, "On Measuring Economic Diversification', A Comment", Land Economics, vol. 54, núm. 1, 1978, pp. 106-109.

Wiener, N., Cybernetics, segunda edición, Cambridge, MIT Press, 1961.

Wold Watch Institute, The State of the World 2005 (L'état de la planète 2005), 2005: http://www.delaplanete.org. 